\title{
Curso 'Uso de evidências na gestão municipal da saúde': uma experiência pioneira
}

\author{
Workshop on the use of evidence in municipal health management: \\ a pioneering experience \\ Taller sobre el uso de la evidencia en la gestión municipal de salud: \\ una experiencia pionera
}

Jorge Otavio Maia Barreto ${ }^{*}$, Nathan Mendes Souza ${ }^{2}$, Carmem Veronica Mendes Abdala ${ }^{3}$,

Ulysses Panisset ${ }^{4}$, Gilvânia de Melo ${ }^{5}$, Rosemeire Rocha Pinto ${ }^{6}$

Palavras-chave: Administração em Saúde

Pública

Uso da Informação Científica na Tomada de Decisões em

Saúde

Sistemas Locais de Saúde Sistema Único de Saúde

Keywords:

Public Health Administration Use of Scientific Information for Health Decision Making Local Health Systems Unified Health System

\section{Resumo}

É apresentada a experiência do curso Uso de evidências na gestão municipal da saúde, realizado durante o XXVI Congresso do CONASEMS com 0 objetivo de promover a incorporação da evidência científica como subsídio do processo de tomada de decisão de gestores e profissionais de saúde. 0 curso foi concebido, planejado e realizado no âmbito das parcerias institucionais da EVIPNet Brasil e seus resultados apontam aspectos relevantes para discussão acerca das estratégias e ferramentas para disseminação e utilização de evidências de pesquisa em saúde na gestão do Sistema Único de Saúde no Brasil.

\section{Abstract}

This paper presents the experience of a workshop on the use of evidence in municipal health management held during the XXVI Congress of CONASEMS that aimed to promote the incorporation of research evidence to inform the decision making process by managers and healthcare providers. The workshop was designed, planned and conducted within EVIPNet Brazil institutional partnerships EVIPNet and its outputs comprised relevant discussion on strategies and tools for dissemination and use of research evidence for the management of the Unified Health System in Brazil. 


\begin{abstract}
Palabras clave: Resumen
Administración en Salud

Pública

Uso da la Información

Científica en la Toma de

Decisiones en Salud

Sistemas Locales de Salud

Sistema Único de Salud

Aquí se presenta la experiencia del taller acerca del uso de la evidencia en la gestión municipal de salud que ocurrió durante el XXVI Congreso de CONASEMS con el objetivo de promover la incorporación de la evidencia de investigación para informar el proceso de toma de decisiones por los directivos y profesionales de la salud. El taller fue diseñado, planificado y llevado a cabo por las asociaciones institucionales de EVIPNet Brasil y sus resultados aportan relevante discusión acerca estrategias y herramientas para la diseminación y utilización de las evidencias de investigación en salud en la gestión del Sistema Único de Salud en Brasil.
\end{abstract}

\section{Introdução}

A tradução do conhecimento científico visa tornar acessíveis as evidências de pesquisa como subsídio na formulação de políticas, gestão de sistemas e serviços de saúde, bem como na tomada de decisão clínica, em especial na Atenção Primária à Saúde (APS). Intervençôes fundamentadas em estratégias e ferramentas da traduçáo do conhecimento - como os esforços para se fazer chegar evidência a determinadas audiências-chave (push), aumentar a demanda por parte de diferentes públicos (user pull) e favorecer o ambiente para o uso de produtos de pesquisa (research climate) e o intercâmbio entre produtores e usuários de pesquisa científica (exchange) - constituem ações desenvolvidas em todo o mundo ${ }^{1-4}$.

No Brasil, várias são as instituições que visam apoiar e incentivar a disseminação e o uso do conhecimento científico para o aprimoramento dos resultados no Sistema Único de Saúde (SUS) mediante a tradução da evidência para a prática, como, dentre outros exemplo, o Conselho Nacional de Secretários Municipais de Saúde (CONASEMS), no âmbito da gestáo local, e a Rede de Pesquisa em APS (www. rededepesquisaaps.org.br), no campo acadêmico e de gestão. Nesse contexto, uma experiência pioneira e colaborativa nascida dentro da EVIPNet Brasil* aponta novos rumos para a disseminação do uso de evidências científicas na gestão de sistemas locais de saúde. Este trabalho se constitui no relato de experiência da concepção, planejamento, execução e avaliação do curso 'Uso de evidência na gestáo municipal de saúde'. Tal iniciativa visou à promoção do uso de evidências científicas como subsídio para a formulação de políticas e tomada de decisão na gestão local do Sistema Único de Saúde (SUS) e

\footnotetext{
* Evipnet: Evidence-Informed Policy Network (Rede para Políticas Informadas por Evidências). Iniciativa promovida pela Organizaçáo Mundial da Saúde (OMS), cujo objetivo é apoiar o desenvolvimento de políticas informadas por evidências científicas, por meio de parcerias entre gestores, representantes do controle social e pesquisadores. No Brasil, são parceiros nessa proposta: Ministério da Saúde/ Departamento de Ciência e Tecnologia (MS/DECIT); Ministério da Saúde/Secretaria de Atenção à Saúde (MS/SAS); Ministério da Saúde/Secretaria de Vigilância da Saúde (MS/SVS); Representaçáo da Organização Pan-Americana da Saúde no Brasil (OPAS); Centro Latino-Americano e do Caribe de Informação em Ciências da Saúde (BIREME/OPAS/OMS); Conselho Nacional de Secretários de Saúde (CONASS); Conselho Nacional de Secretários Municipais de Saúde (CONASEMS); Comissão Intersetorial de Ciência e Tecnologia do Conselho Nacional de Saúde (CICT/ CNS); Associaçáo Brasileira de Pós-Graduação em Saúde Coletiva (ABRASCO); e Fundaçăo Oswaldo Cruz (FIOCRUZ).
}

foi realizada em maio de 2010 durante o XXVI Congresso de Secretarias Municipais de Saúde, em Gramado, Rio Grande do Sul, Brasil.

\section{A proposta do curso 'Uso de evidências na gestão municipal da saúde'}

A iniciativa agregou instituições nacionais e internacionais líderes na promoção do uso do conhecimento científico na clínica, política e gestão da saúde, bem como na promoção do intercâmbio entre diversos atores sociais tais como pesquisadores, políticos, gestores, sociedade civil organizada e usuários de serviços de saúde. Seus objetivos gerais foram: apresentar o uso de evidências científicas como subsídio do processo de tomada de decisão na gestão de serviços e sistemas municipais de saúde, bem como na prática clínica, com especial enfoque na APS; demonstrar como o uso de evidências obtidas pela pesquisa em saúde pode ser incorporado ao processo de tomada de decisão no âmbito da gestão municipal do SUS e apresentar ferramentas de pesquisa e fontes referenciais para a busca de evidências (Quadro 1).

Todas as etapas do curso contaram com a coordenação de um gestor municipal de saúde que representou o CONASEMS e a colaboraçáo de representante do Programa para Tomada de Decisão da Universidade de McMaster, do Programa Internacional da Universidade de Toronto (ambos no Canadá), da BIREME, do DECIT/SCTIE/MS e da Rede EVIPNet Brasil.

O planejamento da atividade teve concepçáo pluralista e colaborativa, com importante participaçáo de todos os atores institucionais. A partir da proposta do CONASEMS de realizar, durante o XXVI Congresso Nacional de Secretarias Municipais de Saúde, atividade para promover a utilização de evidências científicas na tomada de decisão da gestão local da saúde, foram articulados com as instituiçóes parceiras a discussão da metodologia e os conteúdos a serem utilizados no curso. Durante os dois meses de preparaçáo do curso, foram realizadas duas teleconferências para a discussão da 
Quadro 1. Ementa do curso.

\begin{tabular}{cl}
\hline Carga horária & \multicolumn{1}{c}{ 4 horas-aula (realizadas em 5 sessões de 40 minutos, intercaladas por intervalos) } \\
\hline \multirow{2}{*}{ Objetivos } & Compartilhar conceitos de evidência e de política e gestão informada por evidência; \\
& Sensibilizar gestores em saúde quanto à premência do uso de evidência e \\
& Apresentar recursos (fontes de informação, estratégias e contatos nacionais e internacionais) de apoio ao acesso à \\
& evidência. \\
Público-alvo & Secretários municipais de saúde, gestores de serviços de saúde, profissionais da área de planejamento e gestão da \\
Metodologia & saúde. \\
Material & Dramatização de uma situação-problema enfocando a redução da mortalidade perinatal. \\
Avaliação & Apresentações em PowerPoint, diálogo problematizador e vídeo. \\
Próximos passos & Questionário de avaliação do curso (respondido por 35 dos 65 participantes presentes no curso). \\
& Adequação do curso para membros da sociedade civil organizada e usuários do SUS e \\
& Preparação de oficinas para gestores e seus apoiadores nas esferas federal, estadual e municipal.
\end{tabular}

metodologia mais adequada aos conteúdos pactuados e ao perfil da audiência. A distância entre os envolvidos, fator de dificuldade para interação, foi parcialmente contornada pela troca sistemática de e-mails e compartilhamento de documentos.

O principal desafio verificado durante o planejamento do curso esteve relacionado com a necessidade de se obter comunicação eficiente das temáticas abordadas, além de manter o seu envolvimento e atenção da assistência, em especial nos momentos que previam a participação direta do público mediante a condução interativa proporcionada pelos facilitadores. Diante de tal questão, durante a preparação e planejamento do material e fluxo de apresentação, optou-se pela estratégia de problematização dramatizada, na qual os conteúdos são apresentados e discutidos a partir de caso-problema concreto e relevante, diante do qual as temáticas são articuladas de modo a tornar a apresentação dos conteúdos dinâmica, leve e participativa, com o objetivo de manter a qualidade da assistência ao longo das quatro horas de duração do curso.

Duzentos participantes se inscreveram para o curso, esgotando rapidamente as vagas ofertadas pela organização do evento. Entre os inscritos, encontravam-se representados 24/26 (92\%) estados brasileiros. Rio Grande do Norte, Rio Grande do Sul e Minas Gerais foram os estados que tiveram o maior volume de inscriçóes, sendo que $46 \%$ das vagas foram preenchidas por estados da regiáo Nordeste. Quanto às categorias profissionais participantes, os maiores percentuais de inscritos eram enfermeiros ou médicos atuantes na APS (26\%) e gestores municipais de saúde (27\%).

O perfil dos inscritos também foi considerado no planejamento do curso, reforçando o uso de metodologia com potencial interativo nas apresentações, afastando-se, assim, da formalidade das exposiçóes tradicionais. Tendo em conta a opção tomada pelo uso do recurso da dramatizaçáo de situação em que um gestor municipal buscava alternativas para o enfrentamento de um problema de saúde coletiva no âmbito da APS, e visando ampliar ainda mais o interesse do público participante, foi escolhida a temática da mortalidade perinatal e materna relacionada com a hipertensão na gravidez, especificamente a pré-eclâmpsia e a eclâmpsia, problema comumente vivenciado nos serviços e gestáo locais de saúde. A situação-problema apresentada pelo gestor-coordenador da atividade serviu de 'fio-condutor' para as intervençóes de cada colaborador, na seguinte ordem de conteúdos: i) apresentação dos conceitos de evidência e gestão/organização informada por evidências; ii) uso de evidências no processo de tomada de decisão na gestão de sistemas de saúde; iii) fontes de informação e recursos de busca de evidências científicas; iv) iniciativas nacionais, no âmbito do Ministério da Saúde, para o uso de evidências; v) experiência da colaboração Brasil-Canadá no projeto de Aperfeiçoamento da Gestão em Atençáo Primária (Projeto AGAP)**; e vi) a experiência internacional da EVIPNet no suporte ao uso de evidências para a formulaçáo de políticas de saúde e tomada de decisão na gestão, especialmente em países em desenvolvimento. Durante o desenvolvimento da atividade também foram realizadas intervençôes programadas para facilitar a interação e o envolvimento dos participantes, a fim de se manterem o interesse e a atenção, essenciais ao êxito da atividade.

Ao final do curso promoveu-se o lançamento de duas publicaçóes. O primeiro lançamento foi da versão eletrônica em português da coletânea de artigos publicados na revista Health Research Policy and Systems intitulada: 'Ferramentas SUPPORT para a elaboração de políticas de saúde baseadas em evidências (STP)'. Formuladores de políticas e tomadores de decisóes na clínica, serviços e sistemas de saúde bem como pesquisadores da área da traduçáo do conhecimento

\footnotetext{
** Projeto AGAP: http://agapbr.org
} 
e a sociedade civil organizada encontrarão nessa coletânea ferramentas e estratégias desenvolvidas para apoiar o uso de evidência científica de modo a aumentar a efetividade das ações em saúde.

O segundo lançamento foi do resultado preliminar da primeira síntese de evidências para políticas de saúde produzida pelo grupo executivo da EVIPNet Brasil, com a temática da mortalidade perinatal, apontando e analisando a aplicabilidade de opçôes baseadas em evidências para o enfrentamento do problema.

A programação e material utilizado no curso estão disponíveis no endereço da internet http://www.eventos. bvsalud.org/agendas/conasems, incluindo o livro 'Ferramentas SUPPORT para a elaboração de políticas de saúde baseadas em evidências (STP)'. A versão completa dos resultados preliminares da síntese de evidências sobre mortalidade perinatal pode ser acessada na página da EVIPNet Brasil na internet (http://brasil.evipnet.org)

$\mathrm{O}$ uso de evidências científicas como subsídio do processo de tomada de decisão na gestáo local do SUS pode maximizar os benefícios de saúde a serem obtidos com os recursos disponíveis e assegurar o acesso da população a serviços, tecnologias e medicamentos efetivos e seguros, respeitando princípios de equidade, implicando o aumento da efetividade dos sistemas locais, redução dos custos e riscos, além de representar um importante elemento para a informaçáo do controle social.

Para que evidências científicas sejam utilizadas como subsídio do processo de tomada de decisáo da gestáo e prática clínica, é preciso capacitar gestores e profissionais de saúde para a busca de evidências e posicionamento crítico acerca delas, bem como promover a disseminação social pela tradução do conhecimento, aprimorando o acesso à informação qualificada e a mobilização para o engajamento da sociedade para a utilização de evidências no âmbito da gestáo do SUS.

A experiência do curso 'Uso de evidência na gestão municipal de saúde' obteve forte componente de aprendizado para todos os envolvidos e pode tornar-se relevante para a discussão nacional sobre a incorporaçáo do uso de evidências de pesquisa na prática dos gestores do SUS e profissionais de saúde, em especial na APS, sugerindo-se que o formato adotado pode representar uma estratégia amigável para obter maior adesão junto a essas lideranças, facilitando a assimilação dos conteúdos e a sensibilização para a importância da gestão informada por evidências.

A experiência também pode subsidiar propostas para a realização de novos cursos, com maior duração e mais recursos, baseados no conceito pedagógico de aprendizado conjunto de gestores, profissionais de saúde e pesquisadores, facilitando a comunicação entre academia, serviço e gestão, promovendo ganhos de qualidade para todos.

\section{Avaliação do curso}

A avaliaçáo do curso foi relevante para apontar o quanto se atendeu às expectativas dos participantes e aos objetivos propostos, além da adequação da metodologia aplicada. Embora a avaliação do curso tenha sido opcional, 54\% dos participantes (35/65) completaram o questionário, composto por 19 perguntas divididas em quatro partes:

- Parte 1 - sobre o curso, com 11 perguntas para indicação do grau de concordância/satisfaçáo do participante quanto ao material apresentado e utilizado no curso, ao entendimento dos temas abordados no curso e às habilidades adquiridas para o uso, aplicação e avaliação da evidência na gestão da saúde;

- Parte 2 - sobre a abordagem de aprendizagem, com três perguntas sobre a carga horária, a comunicação pré-curso e o material didático oferecido;

- Parte 3-com uma pergunta sobre a estrutura oferecida e o local do curso; e

- Parte 4-quatro perguntas abertas para que os participantes indicassem o que mais e menos gostaram do curso, recomendaçóes para os próximos cursos e intençôes para aplicação do que foi aprendido no curso.

Os participantes responderam 14 perguntas indicando uma opção entre: excelente, muito bom, bom, mediano, aceitável, ruim, muito ruim. A pergunta sobre a carga horária do curso ofereceu as seguintes opçôes de resposta: muito longa, longa, nem longa nem curta, aceitável, ruim e muito ruim.

A avaliação dos participantes mostrou um índice de 81\% (2776 pontos de 3430) de satisfaçáo de atendimento das expectativas e de concordância com as perguntas sobre a relevância, aplicabilidade e habilidade adquirida para o uso de evidências no processo de decisão em saúde. Quanto à carga horária do curso, $60 \%$ dos participantes avaliaram que não foi nem longa nem curta, e outros $40 \%$ avaliaram como curta a duraçáo de quatro horas do curso.

Na pergunta 'o que mais gostou do curso?', os participantes destacaram a dinâmica, a metodologia e os facilitadores do conteúdo do curso. Ficou evidenciado que a proposta metodológica foi aprovada pelos participantes. Dentre as respostas para a pergunta 'o que menos gostou do curso?', foi destacado o tempo de duraçáo da atividade, apontado como curto. Sobre as recomendaçóes dos participantes para os próximos cursos, as respostas indicaram a necessidade de ampliar a carga horária e a divulgação do curso, oferecer material didático impresso e possibilitar, durante o curso, a prática da busca de informaçáo por meio do uso de 
computadores pelos participantes, além de ampliar a participação de gestores.

No tocante à aplicabilidade dos conhecimentos adquiridos no curso, os participantes indicaram que pretendem usar as evidências nos seus processos diários de resolução de problemas de saúde, acessar as fontes de informaçáo divulgadas no curso, melhorar o seu trabalho em equipe e aplicar o conhecimento adquirido nas suas açóes. Os participantes manifestaram também que os conteúdos e a forma de apresentação produziram, pelo menos inicialmente, mudanças na sua postura diante dos problemas cotidianos vividos em seus contextos locais, em especial quanto ao papel do conhecimento científico no processo de tomada de decisão em todos os níveis do sistema de saúde. Um aspecto importante verificado na avaliação do curso foi que, embora dada a heterogeneidade profissional e regional da assistência, as manifestaçôes apresentadas convergiram quanto à mudança de perspectiva sobre o uso cotidiano do conhecimento cientifico em saúde, pouco variando quanto à forte sensibilização para a inserção da evidência como importante subsídio de decisão nos diferentes cenários de gestáo do SUS.

Assim, considerou-se que os objetivos do curso de compartilhar conceitos de evidência e de política e gestão informada por evidência e, especialmente, sensibilizar os participantes para o uso da evidência científica foram plenamente atingidos, produzindo o impacto planejado para este momento formativo.

\section{Próximos passos}

O formato metodológico adotado para aplicação deste curso surgiu da necessidade de sensibilizar os participantes, tornando significativo o conteúdo proposto por meio da dramatização e da adoçáo de um exemplo prático como 'fio condutor'. Este foi um desafio para os colaboradores e, conforme apresentado no resultado das avaliaçóes, um diferencial para os participantes.

De acordo com esta constatação, propostas para o aperfeiçoamento do formato de capacitação utilizado, associando outras atividades, poderiam contribuir para resultados ainda mais satisfatórios:

\section{- Proposta 1:}

Programas de capacitação de média duração (1 a 2 dias) como evento principal para a capacitação de gestores de saúde, com o objetivo de alcançar esse público específico.

Associar à estratégia de dramatização oficinas que possibilitem a discussão em pequenos grupos, incentivando a participação, a apropriação e uso da informação para a construção do conhecimento. Essa atividade ocorreria entre a iv e a v intervenção dos colaboradores. Este é o momento em que o conteúdo apresentado permite subsidiar a construção dessa proposta.

Nesta proposta, os grupos deveriam apresentar, como produto, um plano básico de trabalho a ser utilizado como guia de referência para profissionais na sua área e local de atuação.

Depois dessa exposição haveria um debate entre colaboradores e participantes. A última parte da capacitação traria a apresentação das experiências exitosas no uso de evidências na gestão de sistemas e serviços de saúde.

\section{- Proposta 2:}

Programas de capacitação de curta duração (2 a 4 horas) incluídos nas agendas dos principais eventos com foco na gestão em saúde com o objetivo de alcançar um público mais amplo e heterogêneo.

Adaptação da capacitação para um formato compacto ou similar ao utilizado de acordo com o tempo disponível.

\section{Referências}

1. Lavis JN. How can we support the use of systematic reviews in policymaking? PLoS Med. 2009; 6(11): e1000141. http://dx.doi. org/10.1371/journal.pmed.1000141

2. Hamid M, Bustamante-Manaog T, Dung TV, Akkhavong K, Fu H, Ma Y, et al. EVIPNet: translating the spirit of Mexico. Lancet 2005;366:1758-60. http://dx.doi.org/10.1016/S0140-6736(05)67709-4

3. EVIPNet Americas Secretariat. EVIPNet Americas: informing policies with evidence. Lancet 2008; 372: 1130-1. http://dx.doi.org/10.1016/ S0140-6736(08)61459-2

4. Gray M. Evidence-based health care and public health: how to make decisions about health services and public health. 3rd ed. Edinburgh: Churchill Livingstone; 2009. 